\title{
Multiwavelets applied to metal-ligand interactions: Energies free from basis set errors
}

\author{
Anders Brakestad, ${ }^{1,2}$ Peter Wind, ${ }^{1,2}$ Stig Rune Jensen, ${ }^{1,2}$ Luca Frediani, ${ }^{* 1,2}$ and Kathrin Helen Hopmann $*, 2$ \\ ${ }^{1}$ Hylleraas Centre for Quantum Molecular Sciences and ${ }^{2}$ Department of Chemistry, UiT The \\ Arctic University of Norway, 9037 Troms $\phi$, Norway. \\ Email addresses: luca.frediani@uit.no, kathrin.hopmann@uit.no
}

\begin{abstract}
Transition metal-catalyzed reactions invariably include steps, where ligands associate or dissociate. In order to obtain reliable energies for such reactions, sufficiently large basis sets need to be employed. In this paper, we have used high-precision Multiwavelet calculations to compute the metal-ligand association energies for 27 transition metal complexes with common ligands such as $\mathrm{H}_{2}$, CO, olefins and solvent molecules. By comparing our Multiwavelet results to a variety of frequently used Gaussian-type basis sets, we show that counterpoise corrections, which are widely employed to correct for basis set superposition errors, often lead to underbinding. Additionally, counterpoise corrections are difficult to employ, when the association step also involves a chemical transformation. Multiwavelets, which can be conveniently applied to all types of reactions, provide a promising alternative for computing electronic interaction energies free from any basis set errors.
\end{abstract}




\section{INTRODUCTION}

A large branch of computational chemistry deals with the study of reaction mechanisms. ${ }^{1,2}$ Many of the studied reactions involve metal complexes that throughout the course of the reaction bind or lose a ligand, for example, there may be incoming substrates such as alkenes or hydrogen $\left(\mathrm{H}_{2}\right)$, or leaving ligands such as solvent or product molecules (Scheme 1). ${ }^{3,4,5,6,7,8,9,10}$

The difficulty of contemporary DFT functionals to accurately compute metal-ligand interactions has been highlighted in the literature. ${ }^{11,12,13,14,15,16,17,18,19,20,21,22}$ Many of the reported studies focus on the performance of different DFT functionals and the importance of including dispersion corrections in the computed energies. ${ }^{11,15}$ There is less focus on the importance of choosing an adequate basis set. ${ }^{23,24,25}$

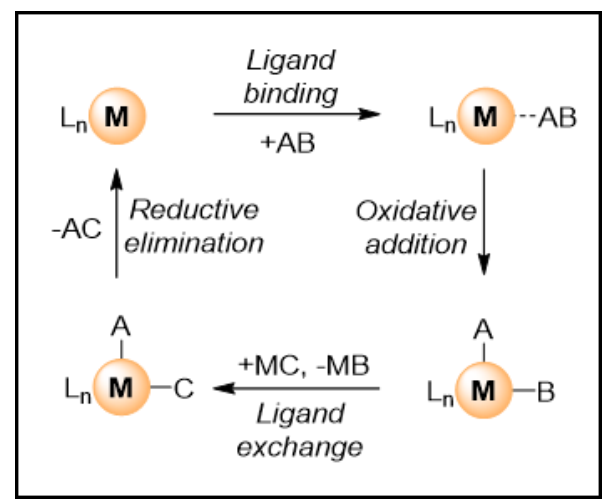

Scheme 1. Generic example of a metal-catalyzed reaction pathway (e.g. a cross-coupling reaction, $M=$ metal) where ligands enter and leave.

The most widely employed basis sets in computational chemistry are based on Gaussiantype orbitals (GTOs). Popular choices include for example Pople and Ahlrichs basis sets. ${ }^{26,27}$ Such basis sets may come in different sizes, with many contemporary studies reporting use of double- $\zeta$ (DZ) and medium-sized triple- $\zeta$ (TZ) basis sets for computing final energies. ${ }^{28,29,30,31,32,33,34,35}$ All available basis sets are in practice finite basis sets, which invariably carry a certain Basis Set Error (BSE), defined as the difference in energy $(E)$ between the complete basis set (CBS) result and the finite basis set (FBS) result.

$$
\mathrm{BSE}=E_{\mathrm{FBS}}-E_{\mathrm{CBS}}
$$

A complete basis set is infinite and therefore a certain level of truncation in the molecular orbital expansion must be accepted for any basis set. This fact is referred to as the 'Basis set truncation problem' and puts very concrete limitations on quantum chemical calculations. It is in addition not possible to know the extent of the BSE for a given basis set, although the variational principle guarantees that enlarging a basis will reduce the BSE. In practical applications of GTOs, users often rely on a favourable cancellation of BSEs, where large errors in absolute energies are partly cancelled, when relative energies (e.g. the energy difference between two states) are computed. 
In presence of a geometrical rearrangement, and in particular when considering energy differences in a reaction, the BSE can be divided into two different (though not completely independent) types of errors: the Basis Set Superposition Error (BSSE) and the remaining Basis Set Incompleteness Error (BSIE). ${ }^{23,25,36}$ The BSSE originates from the fact that atom-centered basis functions follow the nuclear positions. Therefore, the molecular orbitals will be represented by different basis sets when comparing two different geometries, because the basis functions will overlap differently (or in some cases not at all) before and after the geometrical change. ${ }^{37,38,39}$ The BSIE can then be considered as remaining error with respect to the CBS result, although it is important to underline that the two errors cannot be separated completely and both will approach zero in the limit of a CBS.

The most notable example where the BSSE becomes prominent is when two molecules are joined into one model, as illustrated in Figure 1. In this case, the 'borrowing of basis functions' effectively improves the basis set description of the combined molecules compared to the separated molecules, leading to an artificial lowering of the energy.
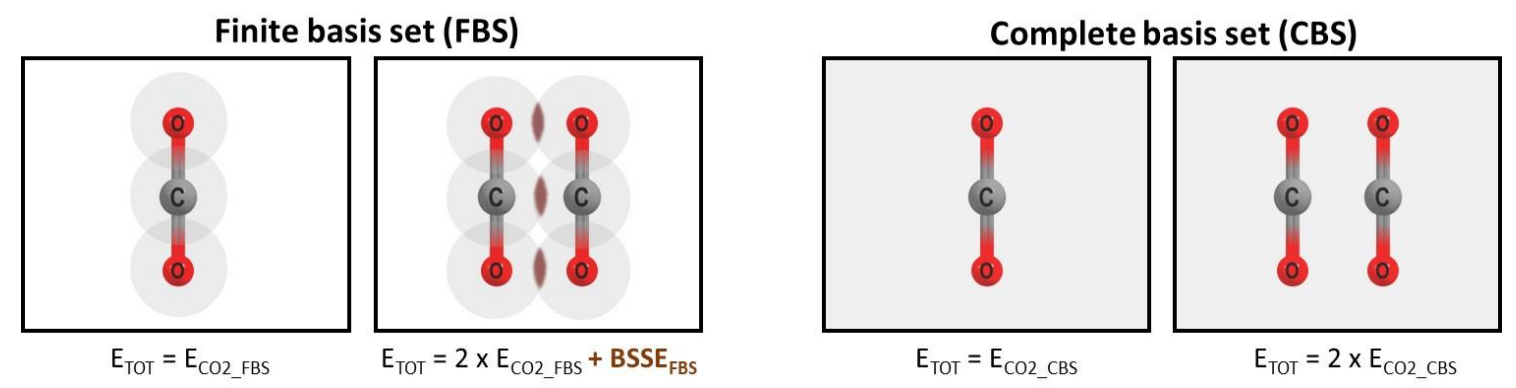

Figure 1. Left: When two molecules or fragments are joined into one computational model, the fragments can steal basis functions from each other, artificially lowering the total energy ( $\left.\mathrm{E}_{\mathrm{tot}}\right)$ of the combined model (an error known as the BSSE). Right: In a complete basis set, the stealing of basis functions does not occur.

A common strategy for dealing with the BSSE is to use the so-called Boys and Bernardi counterpoise (CP) correction. ${ }^{37}$ The $\mathrm{CP}$ correction is often applied for association reactions of noncovalently interacting fragments, ${ }^{25,40,41}$ but is also employed when computing metal-ligand interactions, for example as part of a reaction cycle. ${ }^{14,42,43}$ The theoretical justification for the CP correction has been the subject of much scientific debate since its introduction. ${ }^{38,44,45} \mathrm{~A}$ mathematical proof was published in 1994, which demonstrated that the CP correction eliminates intermolecular BSSEs in simple complexation reactions for full CI (FCI) wave functions. ${ }^{38}$ However, similar theoretical arguments have to our knowledge not been presented for DFT.

The CP correction is typically computed on basis of the complexed system, which is partitioned into fragments, whose energies are computed in presence and absence of the other fragments' basis functions. For non-covalent association and dissociation reactions, the partioning 
is simple, but for reactions where the combination of fragments involves bond-breaking, the partioning becomes ambiguous. As an example, let us consider two reactions; one where $\mathrm{CO}_{2}$ binds to a complex (Scheme 2, left) and one where $\mathrm{CO}_{2}$ is inserted into a metal-ligand bond (Scheme 2, right). For the simple association reaction, the original fragments remain and partitioning is straightforward, but for the insertion reaction, the original fragments no longer exist in the product and it is thus unclear how the system should be partitioned. Reaction types other than simple associations and dissociations are widespread in transition metal-mediated chemistry, such as oxidative additions, reductive eliminations, insertions, transmetallations, and metatheses reactions - for all of these it is not straightforward how to apply a CP protocol.
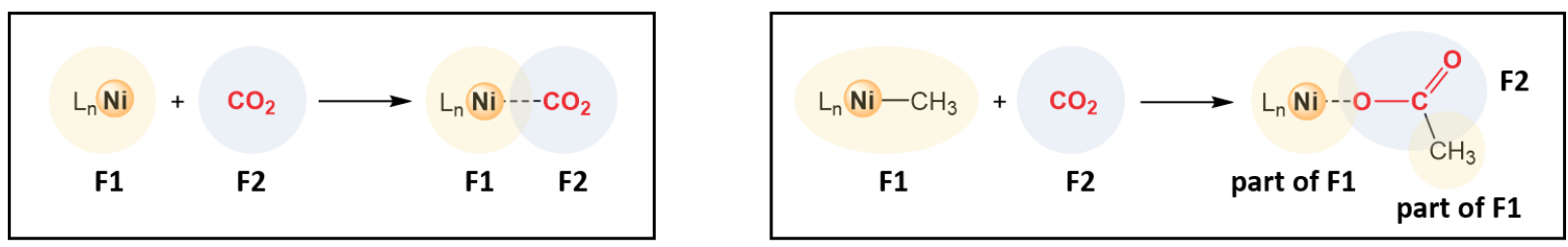

Scheme 2. Left: Simple association reaction, where the complex formed after association easily can be partitioned into the original fragments F1 and F2 in order to compute the BSSE. Right: Association reaction involving a chemical transformation, where the partitioning into original fragments becomes ambiguous and it is unclear how the BSSE should be computed.

Another approach for reducing BSEs is to employ a large basis set. It is a relatively standard procedure in computational studies to perform single-point energy calculations with a larger basis set, using optimized geometries computed with a smaller basis set. In computational organometallic chemistry, such single-point corrections are often carried out with medium-sized TZ basis sets (such as 6-311G(d,p)), ${ }^{31,33,34,35}$ however, the BSE in such basis sets may still be significant (vide infra). Although it is possible to use much larger basis sets (with smaller BSEs) for single point energies, this is rarely done and, as pointed out by Head-Gordon and coworkers, it is remarkably difficult to reach the basis set limit, requiring very large basis sets, such as the quintuple-zeta basis set pc- $4 .^{41}$

In recent years, a new strategy, based on real space methods, ${ }^{46}$ has emerged which can resolve BSE issues in a fundamental and uncontroversial way. In contrast to atom-centered functions, real space methods represent functions as values on a grid. As the representation is fixed in space and does not follow the molecule, the source of BSSE is eliminated. The remaining BSIE can be made arbitrarily small by refining the grid and the method does not rely on cancellation of errors for energy differences, as GTOs do. In this respect, the methods based on Multiresolution Analysis (MRA) ${ }^{47,48,49}$ and Multiwavelets $(\mathrm{MWs})^{50,51,52}$ are particularly attractive: molecular orbitals are represented using polynomials on a predefined grid. Such a grid can be arbitrarily refined by bisection to gain precision. This refinement is fully adaptive, which means that it only takes place where necessary (typically close to the nuclei), thus reducing the computational 
overhead with respect to full-grid methods. The mathematical framework of MRA ${ }^{53}$ guarantees that errors with respect to the basis set limit can be made arbitrarily small in a systematic and predefined way. This simplifies the computational protocol substantially compared to GTO calculations: For GTOs, there is a huge choice of basis sets and it requires experts to fine-tune the basis to the problem at hand, implying that many practitioners fall back to familiar but suboptimal options such as standard double- or triple- $\zeta$ basis sets. With MWs, the only choice to make by the user is the predefined numerical precision requested. Thus, MWs offer a simple protocol, both practically and intellectually, for obtaining energies that are free from basis set errors. We have recently employed MW methods to obtain precise benchmarks on energies, ${ }^{54}$ electric ${ }^{55}$ and magnetic properties. ${ }^{56}$

In this work, we have used multiwavelets to compute the electronic energies for 26 transition metal-mediated reactions, which involve association of common ligands such as $\mathrm{H}_{2}, \mathrm{CO}$, olefins or solvent molecules. To our knowledge, MWs have not been previously used to compute transition metal systems, although it has been suggested that by using them one could improve results for DFT calculations involving metals. ${ }^{57}$ Comparing our Multiwavelet interaction energies to the results obtained with a variety of GTO-type DZ, TZ and QZ basis sets, we show that BSEs in commonly used GTO basis sets can be very large. Interestingly, use of the counterpoise corrections to correct for BSSEs may lead to a significant underbinding for metal-ligand interactions, potentially bringing the corrected value as far from the MW reference value as the uncorrected one.

\section{COMPUTATIONAL DETAILS}

\section{A. Choice of reactions}

The set of association reactions was based on the following criteria: 1) The reactions should be simple association reactions, so that standard counterpoise corrections (CP) could be applied, 2) The complexes should feature $3 d$ transition metals in order to limit the system size and because not every all-electron basis set studied here is available for heavier metals, 3) The incoming ligands should be experimentally relevant and of varying sizes, 4) The nature of the metal-ligand binding should be diverse, 5) All chemical species should have a closed-shell electronic configuration.

Our reaction set includes 26 transition metal-mediated association reactions, with the full list presented in Scheme 3A-C. Four of these reactions, namely $\mathrm{Cr}-\mathrm{CO}, \mathrm{Cr}-\mathrm{H}_{2}, \mathrm{Cr}-\mathrm{alkene}-1$ and $\mathrm{Ni}-\mathrm{CO}$, were chosen from reference 58 (but we note that some of these have been studied much earlier ${ }^{59}$ ) and one, $\mathrm{Fe}-\mathrm{MeOH}$, is related to our previous work on Fe-catalyzed hydrogenation reactions. ${ }^{60}$ Based on the $\mathrm{Cr}$ and $\mathrm{Ni}$ examples, we designed additional reactions involving association of differently sized alkenes (alkene-1 to alkene-6), different solvent molecules (MeCN, THF, $\mathrm{MeOH}, \mathrm{H}_{2} \mathrm{O}$ ) and of common NHC ligands (NHC1, NHC2, Scheme 3). The optimized coordinates of all species are given in the SI. 
One additional $\mathrm{CO}_{2}$ insertion reaction was computed (Scheme 3D) as an example of a reaction, where $\mathrm{CP}$ corrections become ambiguous to compute, as the original fragments are no longer present in the product. This reaction is not included in the averaging of basis sets, but is discussed separately.
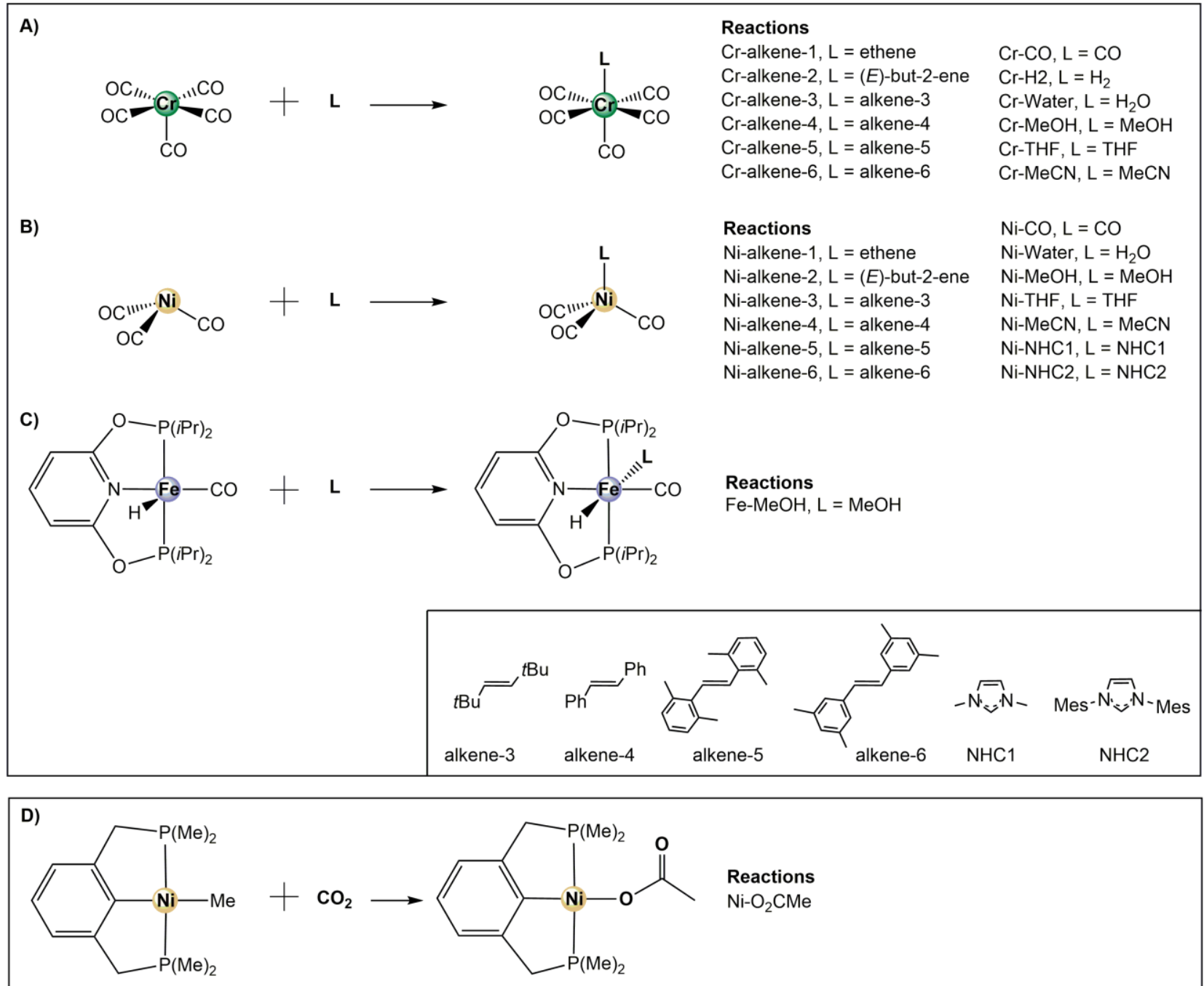

Scheme 3. Overview of the 26 association reactions included in our data set (A-C) as well as one $\mathrm{CO}_{2}$ insertion reaction discussed separately $(\mathbf{D})$.

\section{B. GTO calculations}

All GTO calculations were performed with ORCA ${ }^{61,62}$ versions 4.1.2 and 4.2.1 (see SI, Table S1, for further details) within the restricted Kohn-Sham DFT framework. ${ }^{63,64}$ The SCF cycles were accelerated by the $\mathrm{RI}^{65,66,67,68,69,70,71}$ and $\mathrm{RI}-\mathrm{COSX}$ approximations for GGA and hybrid functionals, respectively. A multigrid scheme was used for the integration grids: Intermediate SCF iterations made use of an angular Lebedev grid of 434 points, and a radial grid of 30, 35, and 40 points for first, second, and third row elements, respectively (as defined by the grid5 ORCA keyword). A final SCF computation was then carried out with a larger angular Lebedev grid of 
590 points, and a radial grid of 40, 45, and 50 points for first, second, and third row elements, respectively (as defined by the finalgrid6 ORCA keyword).

All geometries were optimized in vacuum with the def2-SVP basis set ${ }^{72}$ and included the Grimme's 3rd generation dispersion correction with Becke-Johnson damping functions. ${ }^{73,74}$ For the data presented in the main text, we used the PBE functional, ${ }^{75,76,77}$ however, in the Supporting Information we also present results with BP86 and PBE0. ${ }^{78,79}$ The BP86 and PBE0 results are in close qualitative agreement with PBE (SI, Figures S1 to S12). Default SCF convergence thresholds were used for the geometry optimizations. Geometry convergence criteria were set by the tightopt ORCA keyword, which sets convergence thresholds for the energy change, maximum gradient, RMS gradient, maximum structural displacement, and RMS structural displacement as 1 x $10^{-6}, 1 \times 10^{-4}, 1 \times 10^{-5}, 1 \times 10^{-3}, 1 \times 10^{-4}$, respectively (in atomic units). Finally, a frequency analysis was performed in order to confirm that the optimized structures represented minima on the potential energy surface.

Single-points and counterpoise corrections were performed with ORCA versions 4.1.2 and 4.2.1. SCF convergence was dictated by the tightscf ORCA keywords, which signals convergence if the changes in the total energy and 1-electron energy fall below $1 \times 10^{-8}$ and $1 \times 10^{-5}$, respectively. A range of commonly used GTO basis sets of different sizes were employed in this benchmark study, with examples from Jensen's polarization consistent basis sets, ${ }^{80,81,82,83}$ Ahlrichs' property-optimized def2 basis sets, ${ }^{26}$ Dunning's correlation-consistent basis sets, ${ }^{84,85,86,87}$ Pople's split-valence basis sets $26,88,89,90,91,92,93,94,95$ and a popular combination of Pople basis sets with the LANL2 ECP and accompanying valence basis set. ${ }^{96}$

The GTO basis sets included in this study are as follows:

- Jensen: pc-3, pc-2, pc-1

- Ahlrichs: def2-QZVPP, def-TZVP, def2-SVP

- Dunning: cc-pVQZ, cc-pVTZ, cc-pVDZ

- Pople: 6-311++G(2df,2pd), 6-311G+G(d,p), 6-311G(d,p), 6-31+G(d), 6-31G(d)

(with additional 6-311G and 6-31G results given in the SI, Figure S1-S4).

- 6-311G(d,p) [nonmetals] / LANL2TZ [metals]

\section{Multiwavelet calculations}

All MW calculations were carried out with the free and open-source MRChem quantum chemical software, release version $1 .^{97}$ Information about how to obtain, compile and use the code is available on the documentation web pages. ${ }^{98}$ A computational domain with the size $(-64,64)$ in all three dimensions (angstroms) were used for all molecular systems, with the molecular structure translated such that the center-of-mass was in the origin of the computational domain. A relative precision of $1 \times 10^{-7}$ a. u. (MW7) was used in the generation of our MW data. Two convergence criteria were applied in the SCF optimizations: The change in total energy should be below $1 \times 10^{-}$ 
${ }^{7}$ a.u., and the orbital residuals should be at least $5 \times 10^{-6}$. We remark that the electronic energy is variationally optimized and its error is therefore quadratic in the orbital error. The error threshold of the orbitals should be set to $\sqrt{\varepsilon_{r e l}}$ in order to guarantee that the total energy has been converged to $\varepsilon_{r e l}$. By setting the orbital residual convergence threshold to $50 \varepsilon_{r e l}$, we made a conservative choice in converging the orbital residuals. The SCF procedure was accelerated by the Krylov accelerated inexact Newton procedure. ${ }^{99}$

\section{Internal validation of MW convergence}

Multiwavelet energies represent the CBS limit within the specified precision. When MWs are employed to compute reaction energies, it is important to bear in mind that error cancellation does not take place, when one energy is subtracted from another; instead, one relies on numerical robustness. As a result, care must be taken when two energies (e.g. reactants and products) are subtracted: one must ensure that the number of significant digits is large enough to guarantee that enough precision is retained in the difference. However, for MWs this is a systematic and controllable procedure, as opposed to relying on error cancellation in GTO protocols, whose extent is not known a priori, and which cannot be controlled.

A practical consequence of this is that one must make sure that an appropriate MW precision is used in calculations of individual energies, in order to obtain a sufficient number of significant digits in the interaction energy. We evaluated increasing MW precisions for a subset of the reactions in order to determine the appropriate precision for our data set (Table 1). A low precision of $1 \times 10^{-4}$ (MW4) contains a lot of noise, because of cancellation of significant digits. However, increasing the precision to $1 \times 10^{-6}$ (MW6) yields a precision of minimum $0.1 \mathrm{kcal} / \mathrm{mol}$, with an even higher precision observed for most reactions. For the benchmark data in the main text, we made a conservative choice and used the MW7 interaction energies in our analyses, which our data shows to be correct to approximately $1 \mathrm{cal} / \mathrm{mol}$ for the cases, where we can compare to MW8 data.

Note that the MW validation data presented in Table 1 was computed with BP86, while the benchmark data discussed in the main text was computed with PBE. This discrepancy is due to an unforeseen challenge that arose during data collection: originally all MW and GTO data were computed with BP86, but as we later realized that the BP86 versions in Orca and MRChem are not identical (implying that a comparison of GTO to MW results at the BP86 level would be affected by differences in the implementation of the functional, which our tests indicated could amount to several $\mathrm{kcal} / \mathrm{mol}$, when approaching the CBS limit ), the GTO to MW comparison in the main text was instead based on the PBE functional. We also present a smaller MW validation analysis with the PBE functional in the SI with $1 \times 10^{-5}$ (MW5) and $1 \times 10^{-7}$ (MW7) precisions. The average error of the $1 \times 10^{-5}$ energies compared to the $1 \times 10^{-7}$ reference is $0.0635 \mathrm{kcal} / \mathrm{mol}$, which is close to the error observed with the BP86 functional in Table 1 for the same precision. 
Table 1. Errors in electronic interaction energies $(\mathrm{kcal} / \mathrm{mol})$ computed with increasing $\mathrm{MW}$ precision. We computed all reactions with a precision of $1 \times 10^{-4}$ (MW4), $1 \times 10^{-5}$ (MW5), $1 \times 10^{-6}$ (MW6) and $1 \times 10^{-7}$ (MW7), and a few with $1 \times 10^{-8}$ (MW8). The errors for MW4, MW5, and MW6 were obtained by comparing to MW7 results. The MW7 error was obtained relative to the few MW8 results. With MW6, one obtains at least one correct decimal in the interaction energy, and mostly two or more decimals. With MW7, errors of less than $0.0002 \mathrm{kcal} / \mathrm{mol}$ are obtained, as shown by comparison to the MW8 results.

\begin{tabular}{|c|c|c|c|c|}
\hline Reaction & Error MW4a & Error MW5a & Error MW6a & Error $\mathbf{M W 7}^{\mathrm{b}}$ \\
\hline Cr-Alkene-1 & 0.90779 & 0.02400 & 0.00106 & 0.00007 \\
\hline Cr-Alkene-2 & 1.80526 & 0.03588 & 0.00213 & -0.00003 \\
\hline Cr-Alkene-3 & 2.24401 & 0.15309 & 0.00439 & n.a. \\
\hline Cr-Alkene-4 & 0.21415 & 0.05698 & 0.00508 & n.a. \\
\hline Cr-Alkene-5 & 5.60696 & 0.23589 & 0.00802 & n.a. \\
\hline Cr-Alkene-6 & 2.07854 & 0.15767 & 0.01248 & n.a. \\
\hline Cr-Water & 1.78081 & 0.04122 & 0.00097 & n.a. \\
\hline $\mathrm{Cr}-\mathrm{MeOH}$ & 1.15202 & 0.01078 & -0.00047 & n.a. \\
\hline $\mathrm{Cr}-\mathrm{THF}$ & 2.44000 & 0.09763 & 0.00332 & n.a. \\
\hline $\mathrm{Cr}-\mathrm{MeCN}$ & 1.11360 & 0.07443 & 0.00089 & n.a. \\
\hline $\mathrm{Cr}-\mathrm{CO}$ & 4.10096 & 0.08051 & 0.00371 & n.a. \\
\hline $\mathrm{Cr}-\mathrm{H} 2$ & 1.00121 & 0.04125 & 0.00209 & n.a. \\
\hline Ni-Alkene-1 & -1.13259 & -0.01461 & -0.00086 & 0.00012 \\
\hline Ni-Alkene-2 & 1.69224 & 0.06170 & 0.00208 & 0.00004 \\
\hline Ni-Alkene-3 & -0.06038 & 0.08128 & 0.01371 & n.a. \\
\hline Ni-Alkene-4 & 1.50349 & 0.14009 & 0.00295 & 0.00002 \\
\hline Ni-Alkene-5 & 2.67660 & 0.12706 & 0.00644 & n.a. \\
\hline Ni-Alkene-6 & -0.91128 & 0.06107 & 0.00272 & n.a. \\
\hline Ni-Water & -1.11714 & -0.00497 & 0.00045 & 0.00003 \\
\hline $\mathrm{Ni}-\mathrm{MeOH}$ & -1.04620 & -0.01319 & -0.00048 & 0.00017 \\
\hline Ni-THF & 1.14278 & 0.04561 & 0.00291 & n.a. \\
\hline $\mathrm{Ni}-\mathrm{MeCN}$ & -0.45947 & 0.00010 & 0.00021 & n.a. \\
\hline $\mathrm{Ni}-\mathrm{CO}$ & -0.00376 & 0.01538 & -0.00004 & n.a. \\
\hline Ni-NHC-1 & 0.66576 & 0.06770 & 0.00349 & n.a. \\
\hline Ni-NHC-2 & 3.26662 & 0.48349 & 0.00981 & n.a. \\
\hline $\mathrm{Fe}-\mathrm{MeOH}$ & 1.97012 & -0.13680 & -0.00228 & n.a. \\
\hline Average & 1.25508 & 0.07397 & 0.00326 & 0.00006 \\
\hline
\end{tabular}

n.a. $=$ not available. ${ }^{\mathrm{a} C o m p u t e d}$ as $\Delta E[\mathrm{MWX}]-\Delta E[\mathrm{MW} 7]$, where $\mathrm{X}=4,5,6 .{ }^{\mathrm{b}}$ Computed as $\Delta E[\mathrm{MW} 7]-\Delta E[\mathrm{MW} 8]$.

\section{RESULTS \& DISCUSSION}

Initially we present an analysis of the magnitude of the BSSE with various DZ, TZ and QZ GTO basis sets for the 26 studied transition metal-mediated association reactions. This is followed by an analysis of the effect of the counterpoise (CP) correction - does it bring the GTO results closer to the MW-computed CBS reference value? We then take a closer look at the 6-311G $(\mathrm{d}, \mathrm{p})$ basis set, 
due to its unexpected poor performance. Finally, we show how MWs conveniently can be applied to compute CBS single point energies for insertion reactions.

\section{How large are BSSEs for metal-ligand association reactions?}

It has been reported that the magnitude of the BSSE relative to the non-covalent interaction energy for organic molecules starts off relatively small for minimal basis sets, ${ }^{100}$ then increases as the size of the basis set increases, while it eventually diminishes to negligible magnitudes for very large GTO basis sets. ${ }^{25}$ Medium-sized basis sets of DZ quality provided the largest BSSEs.

We have here computed 26 transition metal-mediated association reactions (Scheme 3) in order to get an overview of how large the BSSE is in these kind of reactions with DZ, TZ and QZ basis sets of different sizes and families. The reactions studied here involve ligands that bind to a metal complex, which are conceptually different from non-covalent interaction energies. We have build our test set to include ligands of various size, many of which are common incoming ligands in metal-catalyzed reactions (such as $\mathrm{H}_{2}, \mathrm{CO}$, alkenes, methanol). ${ }^{3,4,5,6,7,8,9,10}$

Several features are observed from our computed results (Figure 2, see also SI, Figure S1 to S4). Firstly, the magnitude of the BSSE is largest for DZ basis sets, with an average value of $9.92 \mathrm{kcal} / \mathrm{mol}$ for the 26 reactions with the basis set $6-31 \mathrm{G}(\mathrm{d}, \mathrm{p})$. However, the BSSE is also unexpectedly large for the TZ basis set $6-311 \mathrm{G}(\mathrm{d}, \mathrm{p})$, with $8.63 \mathrm{kcal} / \mathrm{mol}$ on average. The combination $6-311 \mathrm{G}(\mathrm{d}, \mathrm{p})$ on non-metal atoms and LANL2TZ on the metal gives a significantly lower average BSSE value of $4.25 \mathrm{kcal} / \mathrm{mol}$ for the 26 reactions, but it is still much larger than the def2-TZVP basis set, with an average BSSE of only $1.05 \mathrm{kcal} / \mathrm{mol}$ (maximum value of 2.19

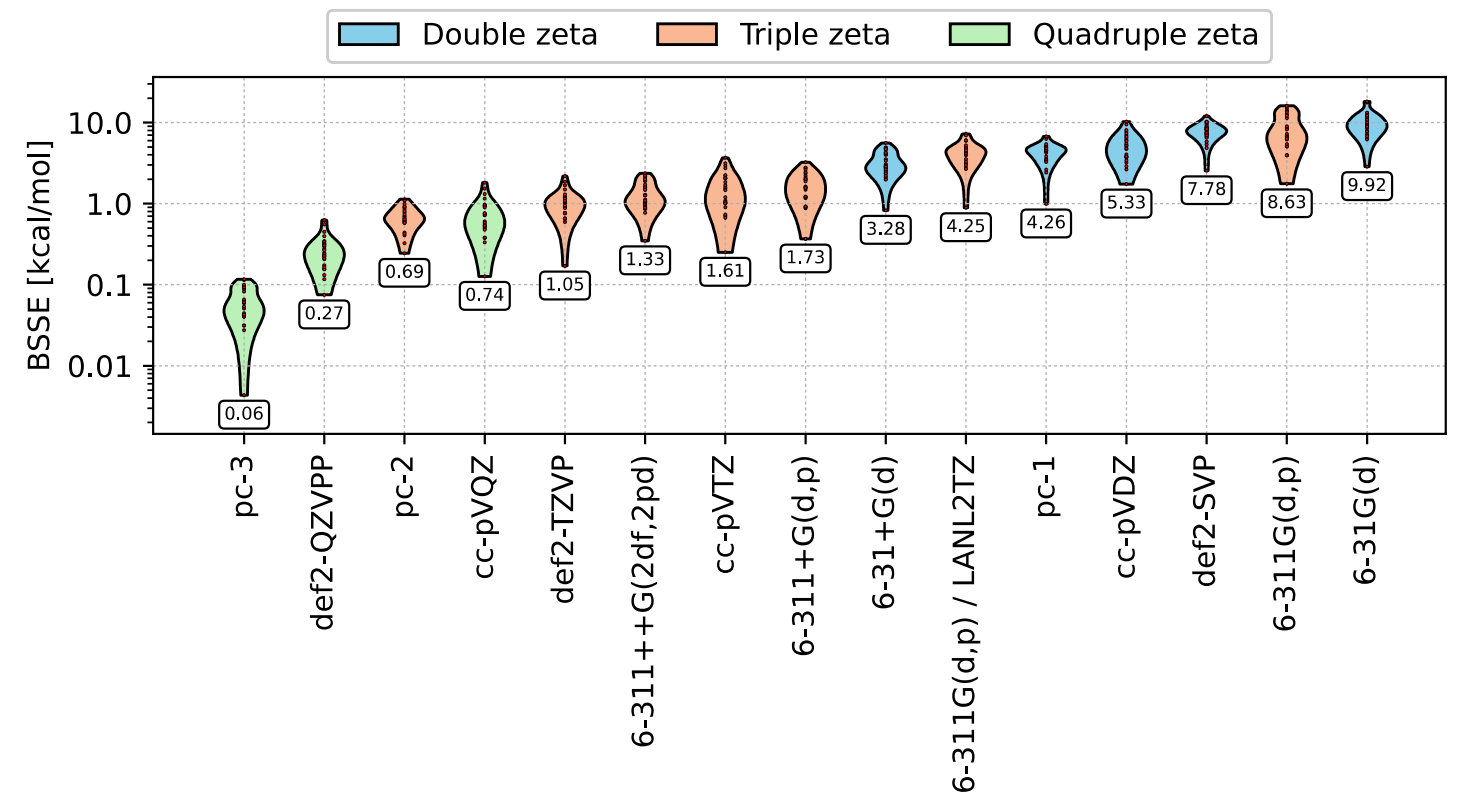

Figure 2. Violin plot summarizing BSSEs (as computed by the counterpoise correction) for selected GTO basis sets (at the PBE level), sorted by ascending averages. The numbers in the inset show the average BSSE ( $\mathrm{kcal} / \mathrm{mol})$ for all association reactions (Scheme 3, A-C) for a given basis set. Additional basis sets are given in the SI, Figure S1. 
$\mathrm{kcal} / \mathrm{mol})$. By comparing all results, it becomes clear that the BSSEs decrease as the zeta-quality increases within each basis set family, but comparing zeta-qualities between families does not follow the same trend. For example, the Jensen triple- $\zeta$ basis set pc-2 has an average BSSE of 0.69 $\mathrm{kcal} / \mathrm{mol}$, which is close to the Dunning-type quadruple- $\zeta$ basis set cc-pVQZ (average error of 0.74 $\mathrm{kcal} / \mathrm{mol})$.

Zooming in on the computed reactions, we see that with almost all basis sets, the largest BSSEs are obtained for the Ni-NHC-2 reaction, which may seem unsurprising, as NHC-2 is the largest ligand studied here. However, there is no clear correlation between the size of the incoming ligand and the BSSE. For example, even the association of a small ligand - such as $\mathrm{CO}$ - can give similar BSSEs as much larger ligands - such as THF - as shown for different TZ basis sets in Figure 3. For DZ and medium-sized $\mathrm{TZ}$ basis sets such as $6-311 \mathrm{G}(\mathrm{d}, \mathrm{p})$, a clear correlation is observed for the type of metal, with BSSEs consistently being larger for the Ni complexes than for the corresponding $\mathrm{Cr}$ complexes (SI, Figure S3). This may be due to the fact that the $\mathrm{Cr}(\mathrm{CO})_{5}$ scaffold is larger than the $\mathrm{Ni}(\mathrm{CO})_{3}$ scaffold, and therefore already has a more complete set of basis functions. For larger basis sets such as def2-TZVP, the BSSEs for the $\mathrm{Cr}$ and Ni systems with the same type of incoming ligand are more similar.

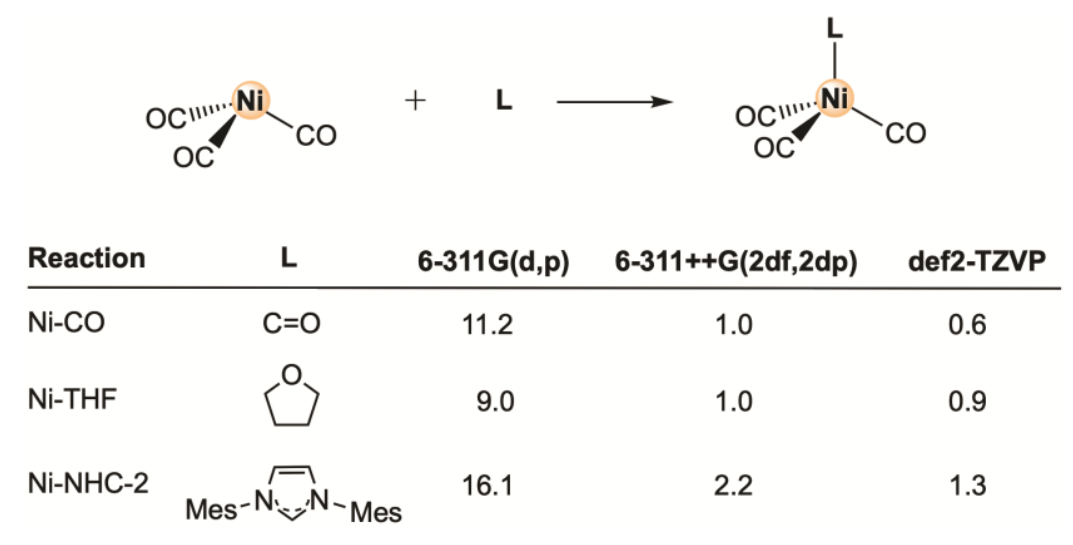

Figure 3. BSSEs (kcal/mol) for three selected reactions with three TZ GTO basis sets (PBE level).

An important point of interest is how large the BSSE is relative to the interaction energy. BSSE proportions of (uncorrected) electronic interaction energies in our test set are presented in Figure 4. For DZ, TZ, and QZ basis sets, the magnitudes of the BSSE are up to approximately 60 $\%, 50 \%$, and $20 \%$ of the electronic interaction energy, respectively. An exception is 6-311G(d,p), for which the BSSE is about $100 \%$ of the interaction energy. Significant variance within each basis set is also observed, spanning at least one order of magnitude for most basis sets. Even interaction energies from large QZ basis sets contain significant proportions of BSSE of up to $20 \%$. 


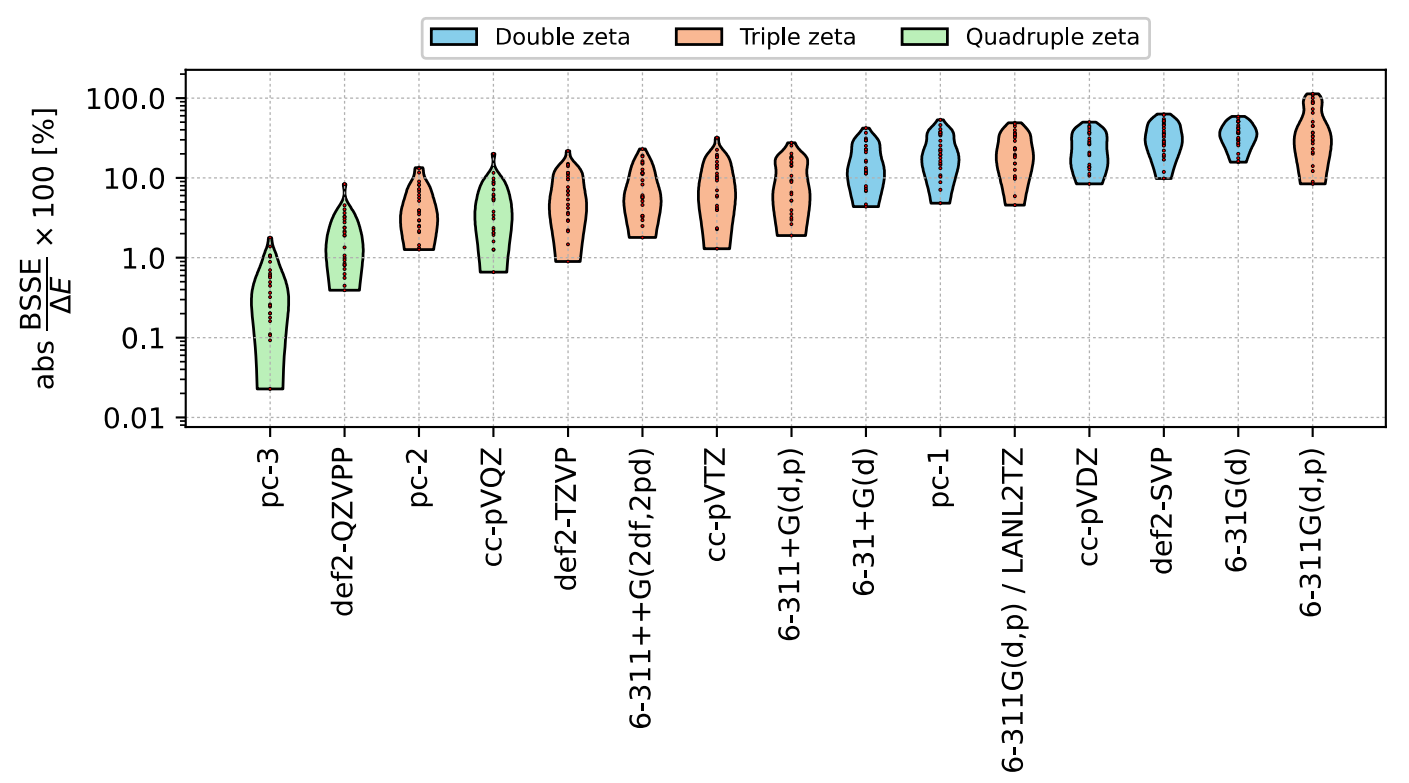

Figure 4. Violin plot summarizing the proportion of electronic interaction energies due to the BSSEs, in percentages, sorted by ascending averages for selected basis sets (PBE level). Additional basis sets are given in the SI, Figure S2.

\section{Can CP corrections bring GTO energies closer to the CBS value?}

We have computed the electronic interaction energies for the 26 reactions in our test set at the complete basis set limit by using a MW basis at high precision $\left(1 \times 10^{-7}=\right.$ MW7, Table 1). With these MW results as a reference, it is possible to gauge how close the uncorrected and CPcorrected GTO energies are to the CBS limit. In the top panel of Figure 5, the GTO basis set errors are plotted on a linear y-axis, in order to show the different signs of corrected and uncorrected interaction energies. It is evident that uncorrected interaction energies tend to approach the CBS limit from below (overbind the complex), while the CP-corrected interaction energies tend to approach from above (underbind the complex). This is in line with other work indicating that including the full $\mathrm{CP}$ correction leads to underbinding. ${ }^{101}$

In the bottom panel of Figure 5, the absolute value of the interaction energies is plotted on a logarithmic y-axis, in order to better show the magnitudes of the errors for corrected and uncorrected interaction energies. Jensen's polarization-consistent basis sets perform the best within each zeta-quality, with the QZ basis set pc-3 delivering deviations from the CBS limit to within approximately $0.1 \mathrm{kcal} / \mathrm{mol}$ or less.

Figure 5 shows that the $\mathrm{CP}$ corrections tend to lower the average error for most basis sets, although there are notable exceptions, such as 6-311G (SI, Figure S13) and 6-311G(d,p) (Figure 5). For these cases, the counterpoise correction does not make the absolute error in the electronic interaction smaller, as the CP-corrected value is as far from the reference value as the CPuncorrected value, just with opposite sign. In order to illustrate how the BSSE and the counterpoise correction may affect the reaction energy of a specific reaction, consider reaction Ni- 

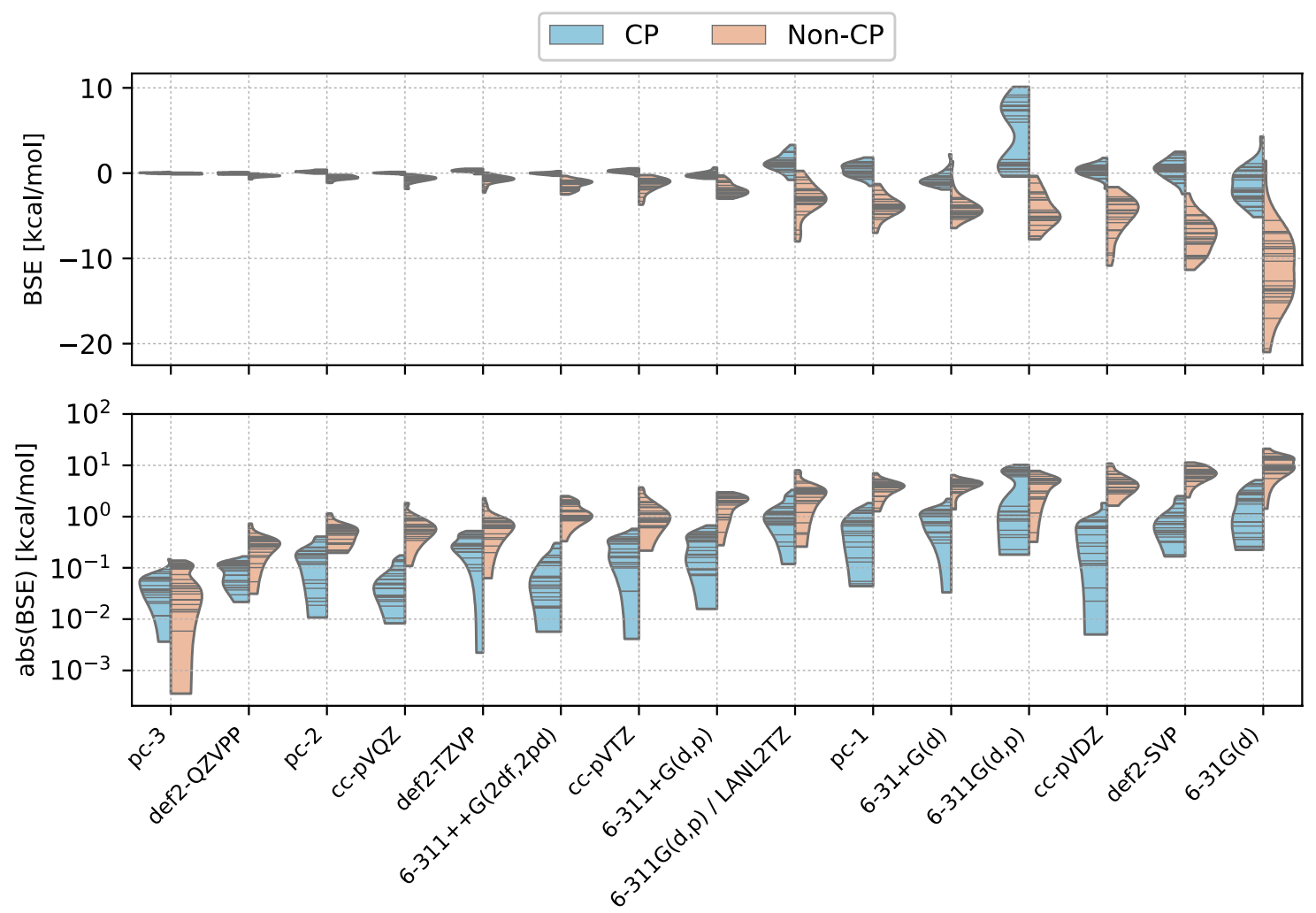

Figure 5. Violin plot summarizing the basis set errors (in $\mathrm{kcal} / \mathrm{mol}$, PBE level) for uncorrected and $\mathrm{CP}$-corrected electronic interaction energies, using our MW7 data as a reference. Top: Basis set errors plotted on a linear axis. Negative values represent overbound complexes. Bottom: Absolute value of basis set errors plotted on a logarithmic axis.

alkene-3 (Scheme 4), which has a medium-sized alkene as incoming ligand (alkenes are typical substrates in metal-catalyzed reactions ${ }^{102}$ ) with a commonly used basis set, 6-311G(d,p). ${ }^{31,33,34,104}$ The electronic association energy computed for this reaction is $-14.0 \mathrm{kcal} / \mathrm{mol}$ with $\mathrm{PBE} / 6-$ $311 \mathrm{G}(\mathrm{d}, \mathrm{p})$ (not including any other corrections). The computed BSSE at the same level is however $15.2 \mathrm{kcal} / \mathrm{mol}$, resulting in an electronic association energy of $+1.2 \mathrm{kcal} / \mathrm{mol}$. The correction (the BSSE) thus has a larger absolute value than the non-corrected electronic interaction energy. Typically, reported computational interaction energies are combined with several correction factors (thermal corrections, ZPVE, entropy corrections, etc.), however, this does not remove the fact that the $\mathrm{CP}$ correction changes the final result by $15.2 \mathrm{kcal} / \mathrm{mol}$. For calculations that desire to approach chemical accuracy $( \pm 1 \mathrm{kcal} / \mathrm{mol})$ a correction factor of this magnitude becomes problematic - unless one can show that the $\mathrm{CP}$ correction brings the electronic interaction energy closer to the value expected for a complete basis set. However, our MW-computed electronic interaction energy for reaction Ni-alkene-3 is $-6.7 \mathrm{kcal} / \mathrm{mol}$, which is approximately in the middle between the uncorrected $6-311 \mathrm{G}(\mathrm{d}, \mathrm{p})$ energy $(-14.0 \mathrm{kcal} / \mathrm{mol}$, error $-7.3 \mathrm{kcal} / \mathrm{mol})$ and the $\mathrm{CP}$ corrected value $(+1.2$, error $+7.9 \mathrm{kcal} / \mathrm{mol})$. This lends some support to previous proposals to use half the counterpoise correction, ${ }^{101,103}$ however, we do note that the underbinding caused by the full CP correction is highly basis set- and ligand-dependent and thus a general reduction of the CP to $50 \%$ is not recommended. 


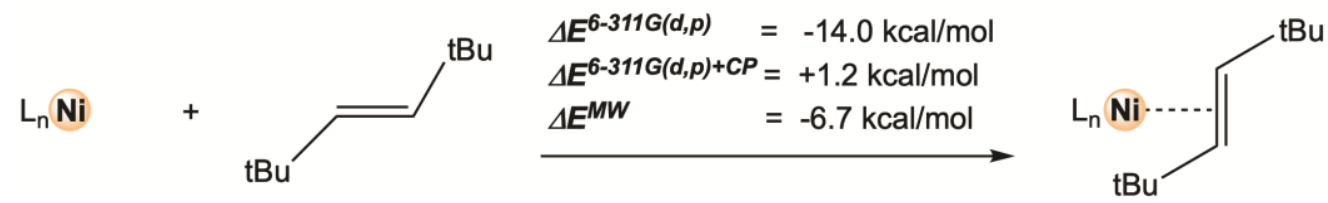

Scheme 4. Reaction Ni-alkene-3 and the 6-311G(d,p) electronic reaction energy with and without CP correction, alongside the complete basis set multiwavelet (MW7) electronic interaction energy (all PBE).

On basis of the overall results in Figures $\mathbf{2}$ to $\mathbf{5}$ we can conclude that on average, BSSEs of 2 to $9 \mathrm{kcal} / \mathrm{mol}$ can be observed for metal-ligand interactions for widely used medium-sized basis sets and that $\mathrm{CP}$ corrections do not consistently improve results. It is important to note that is it impossible to know for a given reaction and a given basis set if the $\mathrm{CP}$ correction will provide improved results or not. A general recommendation may thus be to not use CP corrections, but rather to use larger GTO basis sets for single point energies, or, in order to avoid BSEs altogether, to use MWs.

\section{A closer look at 6-311G(d,p)}

The poor performance of $6-311 \mathrm{G}(\mathrm{d}, \mathrm{p})$ stands out from several of the results presented in the previous sections. It displays BSSEs that more resemble DZ basis sets than TZ basis sets, both in $\mathrm{kcal} / \mathrm{mol}$ and relative to the interaction energy (Figure 2 and 3). Looking at Figure 5, one sees that to a large extent, the $6-311 \mathrm{G}(\mathrm{d}, \mathrm{p}) \mathrm{CP}$ correction leads to an underbinding to about the same extent as the uncorrected values overbind. In other words, one might as well not have performed the correction. Of course, the CP correction's job is not to bring the interaction energy closer to the CBS limit, but rather to remove the BSSE. Whether or not the resulting interaction energy is closer to the CBS limit depends on the interplay between BSSEs and BSIEs. However, the premise for applying the CP correction is that it leads to more robust interaction energies, but this does not seem to be the case for 6-311G(d,p). Table 2 illustrates several examples. For the Cr reactions, the

Table 2. Interaction energies (in $\mathrm{kcal} / \mathrm{mol}$ ) from uncorrected and $\mathrm{CP}$-corrected 6-311G(d,p) calculations, compared to our MW7 reference values (all PBE). The 6-311G(d,p) basis set performs significantly worse than other TZ basis sets, and adding a CP correction does not seem to robustly improve the interaction energies, and even changes the sign of the electronic reaction energy in several cases.

\begin{tabular}{|c|c|c|c|c|c|}
\hline Reaction & $\begin{array}{c}\Delta E \\
6-311 G(d, p)\end{array}$ & $\begin{array}{c}\Delta E \\
\text { 6-311G(d,p) + CP }\end{array}$ & $\begin{array}{c}\Delta E \\
\text { MW7 }\end{array}$ & $\begin{array}{c}\text { BSE } \\
\text { 6-311G(d,p) }{ }^{a}\end{array}$ & $\begin{array}{c}\text { BSE } \\
\text { 6-311G(d,p)+CPa }\end{array}$ \\
\hline Cr-Alkene-1 & -28.1338 & -24.1678 & -24.9850 & -3.15 & +0.82 \\
\hline Cr-Alkene-2 & -25.2397 & -20.1590 & -20.6987 & -4.54 & +0.54 \\
\hline Cr-Alkene-3 & -15.1441 & -8.3921 & -9.6207 & -5.52 & +1.23 \\
\hline Cr-Alkene-4 & -21.1783 & -14.9975 & -15.8985 & -5.28 & +0.90 \\
\hline Cr-Alkene-5 & -15.2103 & -8.3959 & -9.9841 & -5.23 & +1.59 \\
\hline Cr-Alkene-6 & -21.8598 & -15.2904 & -16.3504 & -5.51 & +1.06 \\
\hline Cr-Water & -23.2986 & -17.0040 & -16.6123 & -6.69 & -0.39 \\
\hline Cr-MeOH & -23.3927 & -17.9786 & -18.1595 & -5.23 & +0.18 \\
\hline Cr-THF & -24.1624 & -19.0998 & -19.5419 & -4.62 & +0.44 \\
\hline $\mathrm{Cr}-\mathrm{MeCN}$ & -32.5058 & -28.5472 & -29.4296 & -3.08 & +0.88 \\
\hline
\end{tabular}




\begin{tabular}{|c|c|c|c|c|c|}
\hline $\mathrm{Cr}-\mathrm{CO}$ & -46.6358 & -42.7000 & -43.7583 & -2.88 & +1.06 \\
\hline Cr-H2 & -19.5768 & -17.8168 & -19.0923 & -0.48 & +1.28 \\
\hline Ni-Alkene-1 & -20.6416 & -7.1626 & -16.3267 & -4.31 & +9.16 \\
\hline Ni-Alkene-2 & -16.5817 & -4.5109 & -11.9557 & -4.63 & +7.44 \\
\hline Ni-Alkene-3 & -14.0039 & +1.1945 & -6.6447 & -7.36 & +7.84 \\
\hline Ni-Alkene-4 & -13.9730 & -1.5440 & -8.9230 & -5.05 & +7.38 \\
\hline Ni-Alkene-5 & -12.4233 & +1.6408 & -6.3232 & -6.10 & +7.96 \\
\hline Ni-Alkene-6 & -14.4654 & -1.8928 & -9.1713 & -5.29 & +7.28 \\
\hline Ni-Water & -8.3520 & +0.0439 & -5.9256 & -2.43 & +5.97 \\
\hline Ni-MeOH & -9.3419 & -0.7937 & -7.1498 & -2.19 & +6.36 \\
\hline Ni-THF & -10.3608 & -1.3775 & -8.0951 & -2.27 & +6.72 \\
\hline Ni-MeCN & -16.2620 & -8.0467 & -15.9414 & -0.32 & +7.89 \\
\hline $\mathrm{Ni}-\mathrm{CO}$ & -30.4033 & -19.1056 & -29.2362 & -1.17 & +10.13 \\
\hline Ni-NHC-1 & -41.7440 & -27.6821 & -36.5822 & -5.16 & +8.90 \\
\hline Ni-NHC-2 & -43.8376 & -27.7610 & -36.0936 & -7.74 & +8.33 \\
\hline Fe-МeOH & -21.9143 & -14.6955 & -14.4696 & -7.44 & -0.23 \\
\hline
\end{tabular}

errors of up to $-6.69 \mathrm{kcal} / \mathrm{mol}$ in the $6-311 \mathrm{G}(\mathrm{d}, \mathrm{p})$ interaction energies are reduced to errors of up to $+1.59 \mathrm{kcal} / \mathrm{mol}$ after application of the $\mathrm{CP}$ correction, implying that the results seem reasonable, although a consistent underestimation of the interaction energy (i.e. underbinding) is observed for the $\mathrm{CP}$-corrected values. For the $\mathrm{Ni}$ reactions, the $\mathrm{CP}$-overcorrection is much more severe and in some cases it even reverses the sign of the electronic reaction energy (e.g. Ni-Alkene-3, NiAlkene-5, Ni-Water). The BSE of the CP-corrected energies is between 6 and $10 \mathrm{kcal} / \mathrm{mol}$ for all Ni-reactions. This ill behavior for the Ni reactions is not observed for other basis sets (except 6$311 \mathrm{G}$, which shares the poor performance of $6-311 \mathrm{G}(\mathrm{d}, \mathrm{p}))$, and even the smaller DZ Pople basis sets give more uniform deviations from the CBS reference.

Plotting the BSEs as a function of the number of basis functions used to describe the transition metal complexes (Figure 6), one sees that 6-311G(d,p) indeed should be considered a double-zeta basis set in practice, despite formally being a triple-zeta basis set. The same is
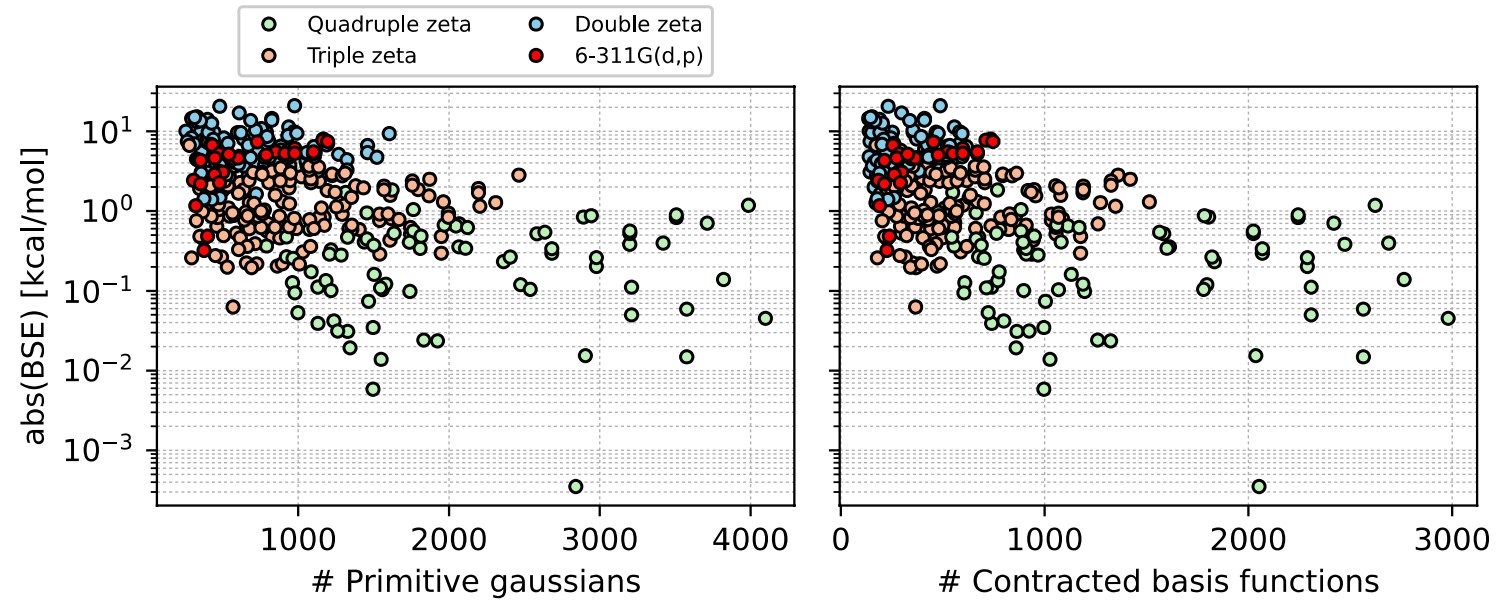

Figure 6. Basis set errors (BSEs) plotted against the number of primitive Gaussian basis functions on the complex (left) and against the number of contracted basis functions of the product complex (right) for all GTO basis sets evaluated here (all PBE). 6$311 \mathrm{G}(\mathrm{d}, \mathrm{p})$ is plotted by itself (in red) and is seen to cluster together with DZ basis sets rather than TZ basis sets with respect to basis set errors and size. 
observed if one instead plots the BSSEs as a function of the basis set size (Figure S15, SI). It can be noted that the 6-311G(d,p) basis set is used in many contemporary studies for computing reaction energies of metal systems..$^{31,33,34,104,105,106}$ On basis of the shortcomings described here, it is strongly recommended to not use this basis set for computing energies.

\section{Convenience of MWs to compute organometallic reaction energies}

The combined results for 26 reactions show that the basis set error in commonly used GTO basis sets can be large (Figure 2 to 6). In order to reduce the BSE, one could use a large GTO basis, such as the QZ basis set pc-3. However, if one desires to quantify the remaining BSSE in large GTO calculations using the counterpoise method, this can become very cumbersome. For example, consider a chemical transformation, such as an insertion into a metal-ligand bond (Figure 7, left). For such instances, it is not straightforward to use the counterpoise correction to compute the BSSE arising from the combination of the fragments in A to give complex C. Unfortunately, in organometallic chemistry, one is very often faced with reaction steps where a change in number of moles occurs simultaneously with a chemical transformation. A possible work-around to compute the $\mathrm{CP}$ is present if an intermediate structure exists, for which the $\mathrm{CP}$ can be computed (B, Figure 7). However, this provides only an approximation of the BSSE present in

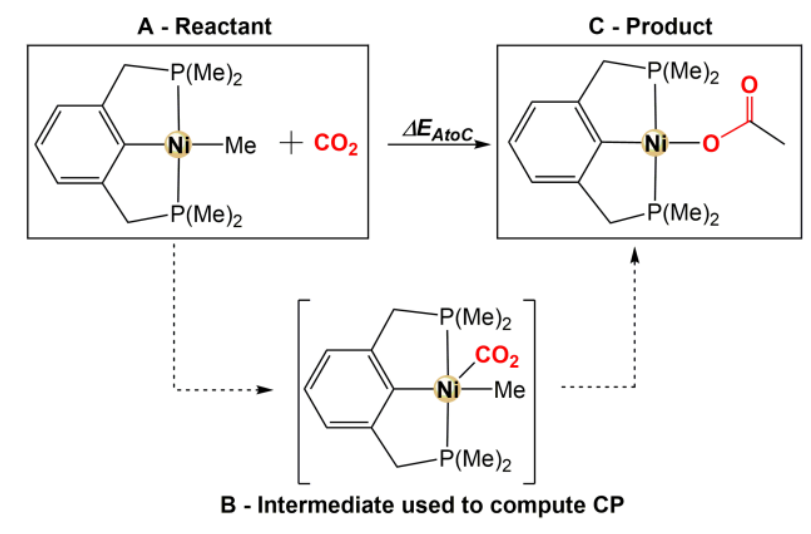

\begin{tabular}{|c|c|c|c|c|}
\hline & $\begin{array}{c}\Delta E \\
\text { Hartree }\end{array}$ & $\begin{array}{c}\Delta E \\
\mathrm{kcal} / \mathrm{mol}\end{array}$ & $\begin{array}{c}\text { BSE } \\
\mathrm{kcal} / \mathrm{mol}\end{array}$ & $\begin{array}{c}\text { Error rel. MW } \\
\%\end{array}$ \\
\hline$\Delta E_{A t o C}^{p c-1}$ & -0.038806 & -24.35 & -3.63 & 17.49 \\
\hline$\Delta E_{A t o C}^{p c-1+C P}$ & -0.035414 & -22.22 & -1.50 & 7.22 \\
\hline$\Delta E_{A t o C}^{p c-2}$ & -0.033179 & -20.82 & -0.09 & 0.46 \\
\hline$\Delta E_{A t o C}^{p c-2+C P}$ & -0.032559 & -20.43 & 0.29 & 1.42 \\
\hline$\Delta E_{\text {AtoC }}^{p c-3}$ & -0.033108 & -20.78 & -0.05 & 0.24 \\
\hline$\Delta E_{A t o C}^{p c-3+C P}$ & -0.033053 & -20.74 & -0.01 & 0.05 \\
\hline$\Delta E_{\text {AtoC }}^{p c-4}$ & -0.033156 & -20.81 & -0.08 & 0.39 \\
\hline$\Delta E_{A t o C}^{p c-4+C P}$ & -0.033155 & -20.80 & -0.08 & 0.38 \\
\hline$\Delta E_{\text {AtoC }}^{M W 6}$ & -0.033028 & -20.73 & - & - \\
\hline
\end{tabular}

Figure 7. Left: Reaction Ni- $\mathrm{O}_{2} \mathrm{CMe}$, involving insertion of $\mathrm{CO}_{2}$ into a Ni-Me bond. In order to compute the $\mathrm{CP}$ correction with GTO basis sets, intermediate B can be used as an approximation, if it exists. Right: Computed electronic reaction energies with pc1 (double- $\zeta$ ), pc-2 (triple- $\zeta$ ), pc-3 (quadruple- $\zeta$ ) and pc-4 (quintuple- $\zeta$ ) GTO basis sets, with and without CP correction (from structure B) compared to MW6 results.

structure $\mathrm{C}$ relative to $\mathrm{A}$. An alternative and straightforward solution is to use MWs instead of large GTOs. In this case, one only has to compute the single point MW energies on states A and C, no additional $\mathrm{CP}$ calculations are needed and no work-around via structure B has to be attempted. Further, the computed results for reaction $\mathbf{N i - O _ { 2 }} \mathbf{C M e}$ indicate that $i$ ) the approximate $\mathrm{CP}$ correction does not consistently reduce the BSE, and $i$ ) the quintuple-zeta basis set pc-4 still has a 
basis set error of $0.4 \%(0.08 \mathrm{kcal} / \mathrm{mol})$ for this reaction (Figure 7, right). While this error is very small with respect to other errors in current DFT calculations, the ultimate goal of computational chemistry must be to compute each quantity as accurately as possible, and to eliminate the need for fortuitous error cancellation. MWs have only recently become able to compute metal systems (and to our knowledge, this manuscript is the first report of MW calculations on transition metal complexes), so their implementation and timings are not yet on par with large GTO basis sets (see

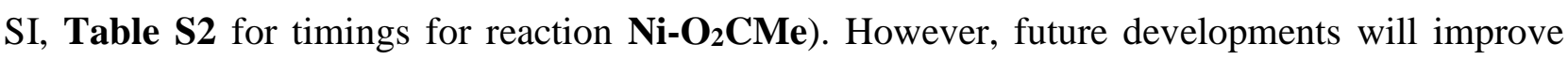
these timings and allow the extension of MW calculations to all elements in the periodic table.

\section{CONCLUSIONS}

We have presented high-precision Multiwavelet (MW) energies for 26 transition metal-mediated reactions involving association of common ligands such as $\mathrm{H}_{2}, \mathrm{CO}$, olefins or solvent molecules (Scheme 1). By comparing to the MW results, we have shown that commonly used DZ and TZ GTO basis sets have large basis set errors. Use of the counterpoise correction to correct for BSSEs leads to underbinding in many cases (Figure 5). A particular poor example is the formally triplezeta basis set 6-311G(d,p), which we show should be considered a double-zeta basis set in practice (Figure 6), and which is not recommended for computing energies.

The results presented here show-case the large variance in electronic interaction energies one can expect for the same reaction step computed with different GTO basis sets. Due to the particular balance of the errors inherent to each basis set, GTO results contain large uncertainties. It is also important to note that reaction steps of different chemical nature may provide very different errors. If one considers the mechanism for a catalytic cycle, each step in the cycle may be chemically distinct (e.g. association, reductive elimination, migratory insertion, metathesis, etc.). A single GTO basis set may not be able to describe each step in the cycle on equal footing, which can lead to unpredictable errors when evaluating relative energies. Thus the computed energy for an intermolecular association step may easily have an error of more than $10 \mathrm{kcal} / \mathrm{mol}$ (as indicated by the large BSSEs observed in our study, Figure 3), but one can expect that a following intramolecular step may have a much smaller error. This type of uncertainty may not be obvious to the non-expert, as it is easy to think of a basis set's description as uniform across different elementary reaction steps.

MWs converge toward the exact CBS limit, to within a precision set by the user. This guarantees a uniform basis set description regardless of the chemical system, implying that MWs conveniently can be applied to any type of reaction. It also eliminates any interplay between the basis set and DFT functional, allowing a user to evaluate a functional's inherent accuracy without considering DFT errors being cancelled by basis set errors. MWs thus constitute a highly promising basis, both for applications to any type of properties, and for use in development of new methodologies, such as new DFT functionals. 


\section{SUPPLEMENTARY MATERIAL}

See the SI (PDF) for results with additional basis sets and DFT functionals, and the XYZ file for optimized coordinates.

\section{DEDICATION}

This paper is dedicated to Ingrid Daubechies, a Belgian physicist and mathematician, whose pioneering work on Wavelets opened new avenues in computational physics and chemistry.

\section{ACKNOWLEDGMENTS}

This work has been supported by a Centre of Excellence Grant (No. 262695), by the Troms $\emptyset$ Research Foundation (No. TFS2016KHH), and by UNINETT Sigma2 through grants of computer time (No. nn9330k, nn4654k). We thank Dr. Diego Garcia-Lopez for the input coordinates of the Ni-pincer complex.

\section{DATA AVAILABILITY}

Additional raw data is openly available at UiT Open Research Data under DOI: https://doi.org/10.18710/WA5YCF. Any other data that support the findings of this study are available from the corresponding authors upon request.

\section{REFERENCES}

\footnotetext{
${ }^{1}$ H. Ryu, J. Park, H. K.Kim, J. Y. Park, S.-T. Kim, and M.-H. Baik, "Pitfalls in Computational Modeling of Chemical Reactions and How To Avoid Them," Organometallics 37, 3228 (2018).

${ }^{2}$ I. Funes-Ardoiz and F. Schoenebeck. "Established and Emerging Computational Tools to Study Homogeneous Catalysis-From Quantum Mechanics to Machine Learning," Chem. 6, 1904 (2020).

${ }^{3}$ G. Sciortino, S. Muñoz-López, A. Lledós, and G. Ujaque, "Comparative Mechanistic Study on the $[\mathrm{Au}(\mathrm{NHC})]^{+}-$Catalyzed Hydration of Alkynes, Alkenes, and Allenes," Organometallics 39, 3469 (2020).

${ }^{4}$ N. X. Gu, P. H. Oyala, and J. C.Peters, " $\underline{H}_{2}$ Evolution from a Thiolate-Bound Ni(III) Hydride," J. Am. Chem. Soc. 142, 7827 (2020).

${ }^{5}$ M. Isegawa, T. Matsumoto, and S. Ogo, "Selective Oxidation of $\mathrm{H}_{2}$ and $\mathrm{CO}$ by NiIr Catalyst in Aqueous Solution: A DFT Mechanistic Study," Inorg. Chem. 59, 1014 (2020).

${ }^{6}$ C. Mealli, G. Manca, R. Tarroni, D. Olivieri, and C. Carfagna, "Computational Overview of a Pd-Catalyzed Olefin Bis-alkoxycarbonylation Process," Organometallics 39, 1059 (2020).

${ }^{7}$ M. Sparta, C. Riplinger, and F. Neese, "Mechanism of Olefin Asymmetric Hydrogenation Catalyzed by Iridium Phosphino-Oxazoline: A Pair Natural Orbital Coupled Cluster Study,” J. Chem. Theory Comput. 10, 1099 (2014).

${ }^{8}$ D. Hong, Y.Shimoyama, Y.Ohgomori, R. Kanega, H.Kotani, T. Ishizuka, Y. Kon, Y. Himeda, and T. Kojima," Cooperative Effects of Heterodinuclear I $^{\mathrm{III}}-\mathrm{M}^{\mathrm{II}}$ Complexes on Catalytic $\mathrm{H}_{2}$ Evolution from Formic Acid Dehydrogenation in Water" Inorg. Chem. 59, 11976 (2020).

${ }^{9}$ Y. Luoa, Z. Huang, Z. Chen, Z. Xu, J. Meng, H.-Y. Li, Q. Meng, and D. Tang, "Strategy Used to Control the Mechanism of Homogeneous Alkyne/Olefin Hydrogenation: AIMD Simulations and DFT Calculations," J. Org. Chem. 85, 11626 (2020)

${ }^{10}$ N. Li, R. Chang, W. Yang, Z. Zhang, and Z. Guo,"Mechanistic Insights into Ni-Catalyzed Difunctionalization of Alkenes Using Organoboronic Acids and Organic Halides: Understanding Remarkable Substrate-Dependent Regioselectivity," Organometallics 39, 2057 (2020).

${ }^{11}$ N. Sieffert and M. Bühl,’Noncovalent Interactions in a Transition-Metal Triphenylphosphine Complex: a Density Functional Case Study" Inorg. Chem. 48, 4622 (2009).

${ }^{12}$ M. Quintal, A. Karton, M. A. Iron, A. D. Boese, and J. M. L. Martin, "Benchmark Study of DFT Functionals for Late-Transition-Metal Reactions," J. Phys. Chem. A 110, 709 (2006).
} 
${ }^{13}$ Y. Minenkov, G.Occhipinti, and V. R. Jensen, “Metal-Phosphine Bond Strengths of the Transition Metals: A Challenge for DFT,” J. Phys. Chem. A 113, 11833 (2009).

${ }^{14} \mathrm{M}$. Sparta, V. R. Jensen, and K. J. Børve, "Accurate metal-ligand bond energies in the $\eta^{2}-\mathrm{C}_{2} \underline{H}_{4}$ and $\eta^{2}-\mathrm{C}_{60}$ complexes of $\mathrm{Pt}\left(\mathrm{PH}_{\underline{3}} \underline{2}_{2}\right.$, with application to their Bis(triphenylphosphine) analogues," Molecular Physics 111, 1599 (2013).

${ }^{15}$ T. Husch, L. Freitag, and M. Reiher, "Calculation of Ligand Dissociation Energies in Large Transition-Metal Complexes," J. Chem. Theory Comput. 14, 2456, (2018).

${ }^{16}$ B. A. Shiekh, "Hierarchy of commonly used DFT methods for predicting the thermochemistry of Rh-mediated chemical transformations," ACS Omega 4, 15435 (2019).

${ }^{17} \mathrm{M}$. A. Iron, and T. Janes, "Evaluating transition metal barrier heights with the latest density functional theory exchange-correlation functionals: The MOBH35 benchmark database," J. Phys. Chem. A. 123, 3761 (2019).

${ }^{18}$ M. Modrzejewski, G. Chalasinski, M. M. Szczesniak, "Assessment of newest meta-GGA hybrids for late transition metal reactivity: Fractional charge and fractional spin perspective," J. Phys. Chem. C. 123, 8047 (2018).

${ }^{19}$ S. Dohm, A. Hansen, M. Steinmetz, S. Grimme, and M. P. Checinski, "Comprehensive thermochemical benchmark set of realistic closed-shell metal organic reactions," J. Chem. Theory Comp. 14, 2596 (2018).

${ }^{20}$ Y. A. Aoto, A. P. de Lima Batista, A. Köhn, and A. G. S. de Oliveira-Filho, "How to arrive at accurate benchmark values for transition metal compounds: Computation or experiment?," J. Chem. Theory Comp. 13, 5291 (2017).

${ }^{21}$ T. Weymuth, E. P. A. Couzijn, P. Chen, and M. Reiher, "New benchmark set of transition-metal coordi- nation reactions for the assessment of density functionals," J. Chem. Theory Comp. 10, 3092 (2014).

${ }^{22}$ M. Steinmetz and S. Grimme, "Benchmark study of the performance of density functional theory for bond activations with (ni,pd)-based transition-metal catalysts," ChemistryOpen 2, 115 (2013).

${ }^{23}$ A. Vidal Vidal, L. C. de Vicente Poutás, O. N. Faza, and C. S. López, “On the Use of Popular Basis Sets: Impact of the Intramolecular Basis Set Superposition Error," Molecules 24, 3810, (2019).

${ }^{24} \mathrm{C}$. Plascencia, J. Wang, and A. K. Wilson, "Importance of the ligand basis set in ab initio thermochemical calculations of transition metal species," Chem. Phys. Lett. 685, 496 (2017).

${ }^{25}$ R. Sure, J. G. Brandenburg, and S. Grimme, "Small Atomic Orbital Basis Set First-Principles Quantum Chemical Methods for Large Molecular and Periodic Systems: A Critical Analysis of Error Sources," ChemistryOpen 5, 94 (2016).

${ }^{26}$ W. J. Hehre, R. Ditchfield, and J. A. Pople, "Self-consistent molecular orbital methods. XII. further extensions of gaussian-type basis sets for use in molecular orbital studies of organic molecules," J. Chem. Phys. 56, 2257 (1972).

${ }^{27} \mathrm{~F}$. Weigend and R. Ahlrichs, "Balanced basis sets of split valence, triple zeta valence and quadruple zeta valence quality for $\mathrm{h}$ to $\mathrm{rn}$ : Design and assessment of accuracy," Phys. Chem. Chem. Phys. 7, 3297 (2005).

${ }^{28}$ K. Lee, J. D. Culpepper, R. Parveen, D. C. Swenson, B. Vlaisavljevich, and S. R. Daly, Modifying Phosphorus(III) Substituents to Activate Remote Ligand-Centered Reactivity in Triaminoborane Ligands," Organometallics 39, 2526 (2020) .

${ }^{29}$ S. Ghorai, and E. D. Jemmis, "DFT Study of C-C and C-N Coupling on a Quintuple-Bonded $\mathrm{Cr}_{2}$ Template: MECP (Minimum Energy Crossing Point) Barriers Control Product Distribution," Organometallics 39, 1700 (2020).

${ }^{30}$ Sakata, S. Shimada, and R. Takeuchi, "Regioselectivity in the Iridium-Catalyzed [2+2+2] Cycloaddition of Unsymmetrical $\alpha, \omega$-Diynes with Nitrile: A DFT Study," Organometallics 39, 2091 (2020).

${ }^{31}$ G. Powers, J. M. Andjaba, M. Zeller, and C. Uyeda, “Catalytic C( $\left.\mathrm{sp}^{2}\right)-\mathrm{H}$ Amination Reactions Using Dinickel Imides,” Organometallics 39, 3794 (2020).

${ }^{32}$ G. Keglevich, R. Henyecz, Z. Mucsi, "Experimental and Theoretical Study on the "2,2'-Bipiridyl-Ni-Catalyzed" Hirao Reaction of $>$ P(O)H Reagents and Halobenzenes: A Ni(0) $\rightarrow \mathrm{Ni}(\mathrm{II})$ or a Ni(II) $\rightarrow \mathrm{Ni}(\mathrm{IV})$ Mechanism?,” J. Org. Chem. 85, 14486 (2020).

${ }^{33}$ M.-S. Xie, B. Huang, N. Li, Y. Tian, X.-X.Wu, Y. Deng, G.-R. Qu, and H.-M. Guo, "Rational Design of 2-Substituted DMAP- $N$-oxides as Acyl Transfer Catalysts: Dynamic Kinetic Resolution of Azlactones, J. Am. Chem. Soc. 142, 19226 (2020).

${ }^{34}$ C. Maquilón, B. Limburg, V. Laserna, D. Garay-Ruiz, J. González-Fabra, C. Bo, M. Martínez Belmonte, E. C. Escudero-Adán, and A. W. Kleij, "Effect of an Al(III) Complex on the Regio- and Stereoisomeric Formation of Bicyclic Organic Carbonates," Organometallics 39, 1642 (2020).

${ }^{35}$ A. Winkler, K. Brandhorst, M. Freytag, P. G. Jones, and M. Tamm, Palladium(II) Complexes with Anionic N-Heterocyclic Carbene-Borate Ligands as Catalysts for the Amination of Aryl Halides, Organometallics 35, 1160 (2016).

${ }^{36} \mathrm{H}$. Kruse, and S. Grimme A geometrical correction for the inter- and intra-molecular basis set superposition error in Hartree-Fock and density functional theory calculations for large systems," J. Chem. Phys. 136, 154101 (2012).

${ }^{37}$ S. Boys and F. Bernardi, "The calculation of small molecular interactions by the differences of separate total energies. Some procedures with reduced errors," Mol. Phys. 19, 553 (1970).

${ }^{38}$ F. B. van Duijneveldt, J. G. C, M. van Duijneveldt-van de Rijdt, and J. H. van Lenthe, "State of the art in counterpoise theory," Chem. Rev. 94, 1873 (1994).

${ }^{39}$ The BSSE is usually divided into two subcategories: Inter- and intramolecular BSSE. While both types originate from the same principles (overlapping basis functions), they differ in the remedies used to correct for them. It is beyond the scope of this paper to review strategies for dealing with intramolecular BSSEs.

${ }^{40}$ B. Brauer, M. K. Kesharwani, and J. M. L. Martin, Some Observations on Counterpoise Corrections for Explicitly Correlated Calculations on Noncovalent Interactions," Chem. Theory Comput. 10, 3791 (2014).

${ }^{41}$ J. Witte , J. B. Neaton, and M. Head-Gordon, "Push it to the limit: Characterizing the convergence of common sequences of basis sets for intermolecular interactions as described by density functional theory," J. Chem. Phys. 144, 194306 (2016).

${ }^{42}$ S. Shafiei-Haghighi, A. Brar, D. K. Unruh, A. F. Cozzolino, and M. Findlater, "Experimental and Computational Studies of Phosphine Ligand Displacement in Iridium-Pincer Complexes Employing Pyridine or Acetonitrile," Organometallics 39, 3461 (2020).

${ }^{43}$ B. L. Oliveira, B. J. Stenton, V. B. Unnikrishnan, C. Rebelo de Almeida, J. Conde, M. Negrão, F. S. S. Schneider, C. Cordeiro, M. G. Ferreira, G. F. Caramori, J. B. Domingos, R. Fior, and G.alo J. L. Bernardes, "Platinum-Triggered Bond-Cleavage of Pentynoyl Amide and $N$-Propargyl Handles for Drug-Activation," J. Am. Chem. Soc. 142, 10869 (2020).

${ }^{44}$ M. Gutowski, J.H. Van Lenthe, J. Verbeek, F.B. Van Duijneveldt, G. Chałasinski, "The basis set superposition error in correlated electronic structure calculations," Chem. Phys. Lett. 124, 370 (1986).

${ }^{45}$ M. M. Szczęśniak and S. Scheiner, "Correction of the basis set superposition error in SCF and MP2 interaction energies. The water dimer," J. Chem. Phys. 84, 6328 (1986).

${ }^{46}$ L. Frediani and D. Sundholm, "Real-space numerical grid methods in quantum chemistry," Phys. Chem. Chem. Phys. 17, 31357, (2015). 
${ }^{47}$ B. Alpert, G. Beylkin, D. Gines, and L. Vozovoi, “Adaptive solution of partial differential equations in multiwavelet bases," J. Comp. Phys. 182, 149 (2002).

${ }^{48}$ B. K. Alpert, “A Class of Bases in $L^{2}$ for the Sparse Representation of Integral Operators," SIAM J. Math. Anal. 24, 246. (1993)

${ }^{49}$ L. Frediani, E. Fossgaard, T. Flå, and K. Ruud, "Fully adaptive algorithms for multivariate integral equations using the non-standard form and multiwavelets with applications to the Poisson and bound-state Helmholtz kernels. in three dimensions" Mol. Phys. 111, 1143 (2013)

${ }^{50}$ R. J. Harrison, G. I. Fann, T. Yanai, Z. Gan, and G. Beylkin, "Multiresolution quantum chemistry: Basic theory and initial applications" J. Chem. Phys. 121, 11587 (2004).

${ }^{51}$ T. Yanai, G. I. Fann, Z. Gan, R. J. Harrison, and G. Beylkin, "Multiresolution quantum chemistry in multiwavelet bases: Hartree--Fock exchange," J. Chem. Phys. 121, 6680 (2004).

${ }^{52}$ T. Yanai, G. I. Fann, Z. Gan, R. J. Harrison, and G. Beylkin, "Multiresolution quantum chemistry in multiwavelet bases: Analytic derivatives for Hartree--Fock and density functional theory," J. Chem. Phys. 121, 2866 (2004).

${ }^{53}$ G. Beylkin, V. Cheruvu, and F. Perez, "Fast adaptive algorithms in the non-standard form for multidimensional problems," Appl. Comput. Harmon. A. 24, 354 (2008).

${ }^{54}$ S. R. Jensen, S. Saha, J. A. Flores-Livas, W. Huhn, V. Blum, S. Goedecker, and L Frediani,“The Elephant in the Room of Density Functional Theory Calculations," J. Phys. Chem. Lett. 8, 1449 (2017).

${ }^{55}$ A. Brakestad, S. R. Jensen, P. Wind, M. D’Alessandro, L. Genovese, K. H. Hopmann, L. Frediani, "Static Polarizabilities at the Basis Set Limit: A Benchmark of 124 Species. J. Chem. Theory Comp. 16, 4874 (2020).

${ }^{56}$ S. R. Jensen, T. Flå, D. Jonsson, R. S. Monstad, K. Ruud, L. Frediani, "Magnetic properties with multiwavelets and DFT: the complete basis set limit achieved," Phys. Chem. Chem. Phys. 18, 21145 (2016).

${ }^{57}$ Á. Vázquez-Mayagoitia, W. S. Thornton, J. R. Hammond, R. J. Harrison, "Quantum Chemistry Methods with Multiwavelet Bases on Massive Parallel Computers," Annu. Rep. Comput. Chem. 10, 3 (2014).

${ }^{58}$ S. Dohm, A. Hansen, M. Steinmetz, S.Grimme, M. P. Checinsk, "Comprehensive Thermochemical Benchmark Set of Realistic Closed-Shell Metal Organic Reactions," J. Chem. Theory Comput.14, 2596 (2018).

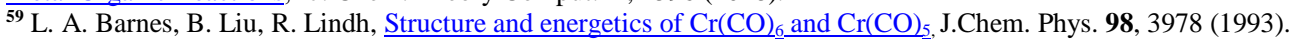

${ }^{60}$ G. Morello. K. H. Hopmann, A Dihydride Mechanism Can Explain the Intriguing Substrate Selectivity of Iron-PNP-Mediated Hydrogenation, ACS Catal. 7, 5847 (2017).

${ }^{61}$ F. Neese, "Software update: the ORCA program system, version 4.0," Wires Comput Mol. Sci. 8:e1327 (2017).

${ }^{62}$ F. Neese, F. Wennmohs, U. Becker, and C. Riplinger, “The ORCA quantum chemistry program package," J. Chem. Phys.152, 224108 (2020).

${ }^{63}$ P. Hohenberg and W. Kohn, "Inhomogeneous electron gas," Phys. Rev. 136, B864 (1964).

${ }^{64} \mathrm{~W}$. Kohn and L. J. Sham, "Self-consistent equations including exchange and correlation effects," Phys. Rev. 140, A1133 (1965)

${ }^{65}$ E. Baerends, D. Ellis, and P. Ros, "Self-consistent molecular Hartree-Fock-Slater calculations I. The computational procedure," Chem. Phys. 2, 41 (1973).

${ }^{66}$ J. L. Whitten, “Coulombic potential energy integrals and approximations," J. Phys. Chem 58, 4496 (1973).

${ }^{67}$ B. I. Dunlap, J. W. D. Connolly, and J. R. Sabin, “On some approximations in applications of x a theory,” J. Chem. Phys. 71, 3396 (1979).

${ }^{68} \mathrm{C}$. V. Alsenoy, "Ab initio calculations on large molecules: The multiplicative integral approximation," J. Comp. Chem. 9, 620 (1988).

${ }^{69} \mathrm{~K}$. Eichkorn, F. Weigend, O. Treutler, and R. Ahlrichs, "Auxiliary basis sets for main row atoms and transition metals and their use to approximate coulomb potentials," Theor. Chem. Acta. 97, 119 (1997).

${ }^{70}$ R. A. Kendall and H. A. Früchtl, "The impact of the resolution of the identity approximate integral method on modern ab initio algorithm development," Theor. Chem. Acta. 97, 158 (1997).

${ }^{71}$ D. E. Bernholdt and R. J. Harrison, "Fitting basis sets for the RI-MP2 approximate second-order many-body perturbation theory method," J. Chem. Phys. 109, 1593 (1998).

${ }^{72} \mathrm{~F}$. Weigend and R. Ahlrichs, "Balanced basis sets of split valence, triple zeta valence and quadruple zeta valence quality for $\mathrm{H}$ to $\mathrm{Rn}$ : Design and assessment of accuracy," Phys. Chem. Chem. Phys. 7, 3297 (2005).

${ }^{73}$ S. Grimme, J. Antony, S. Ehrlich, and H. Krieg, "A consistent and accurate ab initio parametrization of density functional dispersion correction (DFT-d) for the 94 elements H-Pu," J. Chem. Phys. 132, 154104 (2010).

${ }^{74}$ S. Grimme, S. Ehrlich, and L. Goerigk, "Effect of the damping function in dispersion corrected density functional theory," J. Comp. Chem. 32, 1456 (2011).

${ }^{75}$ J. P. Perdew, "Erratum: Density-functional approximation for the correlation energy of the inhomogeneous electron gas," Phys. Rev. B 34, 7406 (1986).

${ }^{76}$ J. P. Perdew, "Density-functional approximation for the correlation energy of the inhomogeneous electron gas," Phys. Rev. B 33, 8822 (1986).

${ }^{77}$ A. D. Becke, "Density-functional exchange-energy approximation with correct asymptotic behavior," Phys. Rev. A 38, 3098 (1988).

${ }^{78} \mathrm{C}$. Adamo and V. Barone, "Toward reliable density functional methods without adjustable parameters: The PBE0 model,” J. Chem. Phys. 110, 6158 (1999).

${ }^{79}$ M. Ernzerhof and G. E. Scuseria, “Assessment of the Perdew-Burke-Ernzerhof exchange-correlation functional,” J. Chem. Phys 110, 5029 (1999).

${ }^{80}$ F. Jensen, "Polarization consistent basis sets: Principles," J. Chem. Phys. 115, 9113 (2001).

${ }^{81}$ F. Jensen, "Polarization consistent basis sets: II. Estimating the Kohn-Sham basis set limit," J. Chem. Phys. 116, 7372 (2002).

${ }^{82}$ F. Jensen, "Polarization consistent basis sets. III. The importance of diffuse functions," J. Chem. Phys. 117, 9234 (2002).

${ }^{83}$ F. Jensen and T. Helgaker, "Polarization consistent basis sets. V. The elements Si-Cl," J. Chem. Phys. 121, 3463 (2004).

${ }^{84}$ T. H. Dunning, "Gaussian basis sets for use in correlated molecular calculations. i. the atoms boron through neon and hydrogen," J. Chem. Phys. 90, 1007 (1989).

${ }^{85}$ D. E. Woon and T. H. Dunning, "Gaussian basis sets for use in correlated molecular calculations. III. The atoms Aluminum through Argon," J. Chem. Phys. 98, 1358 (1993).

${ }^{86}$ N. B. Balabanov and K. A. Peterson, "Systematically convergent basis sets for transition metals. i.Aall- electron correlation consistent basis sets for the 3d elements Sc-Zn,” J. Chem. Phys. 123, 064107 (2005).

${ }^{87}$ N. B. Balabanov and K. A. Peterson, "Basis set limit electronic excitation energies, ionization potentials, and electron affinities for the $3 \mathrm{~d}$ transition metal atoms: Coupled cluster and multireference methods," J. Chem. Phys. 125, 074110 (2006).

${ }^{88}$ R. Krishnan, J. S. Binkley, R. Seeger, and J. A. Pople, "Self-consistent molecular orbital methods. XX. A basis set for correlated wave functions," J. Chem. Phys. 72, 650 (1980). 
${ }^{89}$ A. D. McLean and G. S. Chandler, "Contracted gaussian basis sets for molecular calculations. i. second row atoms, z=11-18," J. Chem. Phys. 72, . 5639 (1980).

${ }^{90}$ M. M. Francl, W. J. Pietro, W. J. Hehre, J. S. Binkley, M. S. Gordon, D. J. DeFrees, and J. A. Pople, "Self-consistent molecular orbital methods. XXIII. a polarization-type basis set for second-row elements," J. Chem. Phys. 77, 3654 (1982).

${ }^{91}$ T. Clark, J. Chandrasekhar, G. W. Spitznagel, and P. V. R. Schleyer, "Efficient diffuse function- augmented basis sets for anion calculations. III. The 3-21+g basis set for first-row elements, Li-F," J. Comp. Chem. 4, 294 (1983).

${ }^{92}$ M. J. Frisch, J. A. Pople, and J. S. Binkley, "Self-consistent molecular orbital methods 25. supplementary functions for gaussian basis sets," J. Chem. Phys. 80, 3265 (1984).

${ }^{93}$ L. A. Curtiss, M. P. McGrath, J.-P. Blaudeau, N. E. Davis, R. C. Binning, and L. Radom, "Extension of Gaussian-2 theory to molecules containing third-row atoms Ga-Kr," J. Chem. Phys. 14, 6104 (1995).

${ }^{94}$ J.-P. Blaudeau, M. P. McGrath, L. A. Curtiss, and L. Radom, "Extension of Gaussian-2 (G2) theory to molecules containing third-row atoms K and Ca," J. Chem. Phys. 107, 5016 (1997).

${ }^{95}$ V. A. Rassolov, J. A. Pople, M. A. Ratner, and T. L. Windus, “6-31G* basis set for atoms K through Zn,” J. Chem. Phys. 109, 1223 (1998).

${ }^{96} \mathrm{P}$. J. Hay and W. R. Wadt, "Ab initio effective core potentials for molecular calculations. potentials for K to Au including the outermost core orbitals," J. Chem. Phys. 82, 299 (1985).

${ }^{97}$ R. Bast, M. Bjorgve, R. Di Remigio, A. Durdek, L. Frediani, G. Gerez, S. R. Jensen, J. Juselius, R. Monstad, and P. Wind, "MRChem: MultiResolution Chemistry," https://doi.org/10.5281/zenodo.4306059 (2020).

${ }^{98}$ MRChem Documentation, https://mrchem.readthedocs.io/en/latest (2020).

${ }^{99}$ R. J. Harrison, "Krylov subspace accelerated inexact newton method for linear and nonlinear equations," J. Comp. Chem 25, 328 (2003).

${ }^{100}$ If there are few basis functions, these are less likely to overlap, which is the reason for the small BSSE of minimal basis sets. Note that a small BSSE does not imply a good basis set, as the overall BSE will be large for minimal basis sets.

${ }^{101}$ K. S. Kim, P. Tarakeshwar, J. Y. Lee, "Molecular Clusters of $\pi$-Systems: Theoretical Studies of Structures, Spectra, and Origin of Interaction Energies," Chem. Rev. 100, 4145 (2000).

${ }^{102}$ J. Diccianni, Q. Lin, and T. Diao, "Mechanisms of Nickel-Catalyzed Coupling Reactions andApplications in Alkene Functionalization," Acc. Chem. Res. 53, 906 (2002)

${ }^{103}$ L. A. Burns, M. S. Marshall, and C. D. Sherrill, "Comparing Counterpoise-Corrected, Uncorrected, and Averaged Binding Energies for Benchmarking Noncovalent Interactions," J. Chem. Theory Comput. 10, 49 (2014).

${ }^{104}$ M. Reiners, A. C. Fecker, D. Baabe, M. Freytag, P. G. Jones, and M. D. Walter,“Cobalt and Nickel Compounds with Pentadienyl and EdgeBridged Pentadienyl Ligands: Revisited", Organometallics 38, 4329 (2019).

${ }^{105}$ R. Jain, A. Mamun, R. Buchanan, P. M. Kozlowski, and C.A.Grapperhaus, "Ligand-Assisted Metal-Centered Electrocatalytic Hydrogen Evolution upon Reduction of a Bis(thiosemicarbazonato)Ni(II) Complex", Inorg. Chem. 57, 13486 (2018).

${ }^{106}$ R. Mondol, and E. Otten, "Aluminum Complexes with Redox-Active Formazanate Ligand: Synthesis, Characterization, and Reduction Chemistry", Inorg. Chem. 58, 6344 (2019). 